\title{
Perspective taking eliminates differences in co-representation of out-group members' actions
}

\author{
Barbara C. N. Müller · Simone Kühn • \\ Rick B. van Baaren · Ron Dotsch • Marcel Brass • \\ Ap Dijksterhuis
}

Received: 23 November 2010/ Accepted: 22 March 2011/Published online: 5 April 2011

(C) The Author(s) 2011. This article is published with open access at Springerlink.com

\begin{abstract}
Coordinated action relies on shared representations between interaction partners: people co-represent actions of others in order to respond appropriately. However, little is known about the social factors that influence shared representations. We investigated whether actions performed by in-group and out-group members are represented differently, and if so, what role perspective-taking plays in this process. White participants performed a joint Simon task with an animated image of a hand with either white or black skin tone. Results of study I demonstrated that actions performed by in-group members were co-represented while actions of out-group members were not. In study II, it was found that participants co-represented actions of out-group members when they had read about an out-group member and to take his perspective prior to the actual experiment. Possible explanations for these findings are discussed.
\end{abstract}

Keywords Joint action - Action co-representation . Action-perception link · Task sharing · Group membership

\section{Introduction}

Observing the actions of others leads to automatic activation of motor representations in the observer (Brass et al. 2001; Dijksterhuis and Bargh 2001; Sebanz et al. 2003, 2005; Prinz 1997), a phenomenon termed action co-representation.

B. C. N. Müller $(\bowtie) \cdot$ R. B. van Baaren · R. Dotsch ·

A. Dijksterhuis

Behavioural Science Institute, Radboud University Nijmegen,

PO Box 9104, 6500 HE Nijmegen, The Netherlands

e-mail: B.Muller@psych.ru.nl

S. Kühn $\cdot$ M. Brass

Department of Experimental Psychology,

Ghent University, 9000 Ghent, Belgium
Co-representation of actions is an essential process for successful social interaction, supporting the understanding of others' actions, goals, and feelings of others (e.g., Preston and de Waal 2002). Recent research demonstrated that representing others' actions is not as universally applicable as previously suggested, but that higher-order processes can have a strong impact on shared action representations. For instance, co-representation only occurs when the interaction partner is perceived as an intentional agent (Müller et al. 2011; Tsai et al. 2008). Furthermore, it has been shown that the valence of interpersonal interactions has a strong impact on how people represent others and their actions (Hommel et al. 2009). In their study, Hommel and colleagues demonstrate that only positive interactions lead to action corepresentation, while negative interactions between jointly acting participants eliminated co-representation of action. The authors suggested that action co-representation is a flexible phenomenon that depends on social factors.

One of the most important social factors in human social interactions is group membership. According to Social Identity Theory (Tajfel and Turner 1979), people derive a substantial part of their self-concept from membership in social groups. It has been shown that out-group members are evaluated more negatively than in-group members (Brewer 1979), and that self-other overlap increases when people belong to the same group (e.g., Aron et al. 2005). Prior literature already demonstrated a modulation of error-related brain activity during observation of actions performed by in-group versus out-group members in a competitive context (Newman-Norlund et al. 2009), and differences in motor cortex activation during observation of in-group versus out-group members' actions were found (Gutsell and Inzlicht 2009). Our first study tries to extend these findings by showing that even outside a competitive context, actions performed by out-group members are 
co-represented less than actions performed by in-group members. Furthermore, as little is known about how fixed this moderation of co-representation by social factors may be, study II was conducted to explore whether the influence of social factors is flexible, and whether this can be manipulated by perspective taking.

In both studies, we used the joint Simon task to measure action co-representation (Hommel et al. 2009; Sebanz et al. 2003; Tsai and Brass, 2007; Tsai et al. 2006). In the classical Simon task, participants respond to the colour of a stimulus (e.g., pressing a left key when a red stimulus appears or pressing a right key when a green stimulus appears on the screen) while ignoring the spatial location of the stimulus. The Simon effect refers to the finding that participants respond slower when the spatial relationship between stimulus and response is incompatible (e.g., pressing a left key in response to a stimulus on the right) than when it is compatible (e.g., pressing a left key in response to a stimulus on the left). The typical Simon effect disappears when participants respond only to one stimulus colour (e.g., to the green stimuli with a right response but not to the red stimuli) in a go/no-go version of the task. Probably, the reason is that a left response is only coded as left when it is encoded in a context in which there are meaningful right responses on the right. The Simon effect reappears when participants perform the task jointly with another person carrying out the alternative response (Sebanz et al. 2003). In a social setting, participants seemingly co-represent the action of interaction partners in a manner that leads to a reappearance of the Simon effect.

In the first study, white participants performed a joint Simon task, co-acting with either a white in-group member or a black out-group member. We expected that the actions of an in-group member would be co-represented, whereas actions of out-group members would not be co-represented.

\section{Study I}

Methods

\section{Participants}

Twenty-seven white students from the Radboud University Nijmegen participated in this experiment ( 22 women; 17-26 years). They received course credit or financial compensation for their participation. All participants were right-handed and had normal or corrected-to-normal vision.

\section{Experimental design, materials and procedure}

The experiment consisted of a 2 (hand colour: white vs. black) $\times 2$ (compatibility: compatible vs. incompatible) repeated-measures design, with hand colour and compatibility as within-subject factors. All participants performed the same joint Simon task, which is essentially a go/no-go task. They put their right hand on the righthanded side of a 17 LCD screen, where a single response key was located. At the centre of the screen, the standard Simon stimulus was presented, a rectangle surrounding three horizontally arranged circles. In each trial, one of the circles was coloured either green or red. In all conditions, an image of either a white or black left hand was displayed along the left-hand side of the screen in each trial. These hands differed only in skin colour and were matched for shape and size (Fig. 1). Participants were instructed to press the response key with their right hand whenever a red dot appeared on the screen, regardless of the position of the dot. The hand displayed along the left side 'responded' to green dots, thus pressing a button on participants' no-go trials. A five-frame image sequence (38 ms per frame) was presented, showing the hand in a series of postures with the index finger approaching the response button. The first image of this sequence was used as a fixation display.

No-go trials were preceded by a $500-\mathrm{ms}$ fixation display. A green target was then presented for $150 \mathrm{~ms}$. After a variable interval (300-450 ms), the image sequence started. Lastly, a fixation display was presented (1,000 ms). Go trials also started with a 500-ms fixation display. Next, a red target was presented for $150 \mathrm{~ms}$. RTs were measured from the onset of the target. Participants were instructed to respond for red targets as quickly as possible, without making too many errors. Response times (RTs) were recorded from the onset of the target. Participants were instructed to respond to the red targets as quickly as possible, without making errors.

The experiment included 45 go trials and 45 no-go trials for both the white and the black hand conditions ( $20 \mathrm{go} /$ compatible trials; 20 go/incompatible trials; 5 go/neutral trials where the target was presented in the centre position). All participants performed two blocks of the joint Simon task, one with a white hand and one with a black hand. The order of these blocks was counterbalanced across subjects. To prevent carry-over effects between conditions, a 2-min break between blocks was employed.

\section{Results and discussion}

All trials with reaction times (RTs) above $1,500 \mathrm{~ms}$ or below $150 \mathrm{~ms}$ ( 3 trials, $0.1 \%$ ) were excluded from further analyses. RTs were log-transformed but untransformed RTs are reported in ms. Difference scores were calculated by subtracting the average RT of compatible trials from the average RT of incompatible trials. Two participants with 


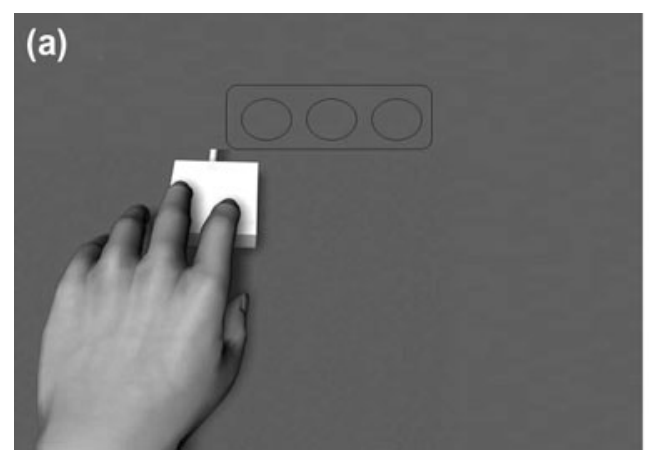

Fig. 1 Sample frames of the white hand (a) and black hand (b)

difference scores 2.5 SD's above the mean were excluded from further analysis.

A 2 (hand colour: white vs. black) $\times 2$ (compatibility: compatible vs. incompatible) GLM repeated-measures analysis revealed a significant main effect of compatibility; participants were slower on incompatible trials $(M=379$; $\mathrm{SE}=10)$ than on compatible trials $(M=369 ; \mathrm{SE}=10)$, $F(1,24)=15.36, P=.001, \eta \rho^{2}=.39$. The main effect of hand colour was not significant, $F(1,24)=1.95, P=.18$. Furthermore, a significant interaction between hand colour and compatibility was obtained, $F(1,24)=4.82, P=.04$, $\eta \rho^{2}=.17$ (Fig. 2). Bonferroni-corrected post hoc comparisons revealed that with a co-acting white agent, participants showed the expected compatibility effect, with slower RTs for incompatible trials $(M=385 ; \mathrm{SE}=9)$ than on compatible trials $(M=370 ; \mathrm{SE}=9), F(1,24)=$ $19.08, P=.001, \eta \rho^{2}=.44$. However, no compatibility effect occurred with a co-acting black agent, $F(1,24)=$ $2.04, P=.17$.

In sum, whereas participants were faster for compatible than on incompatible trials when interacting with a white

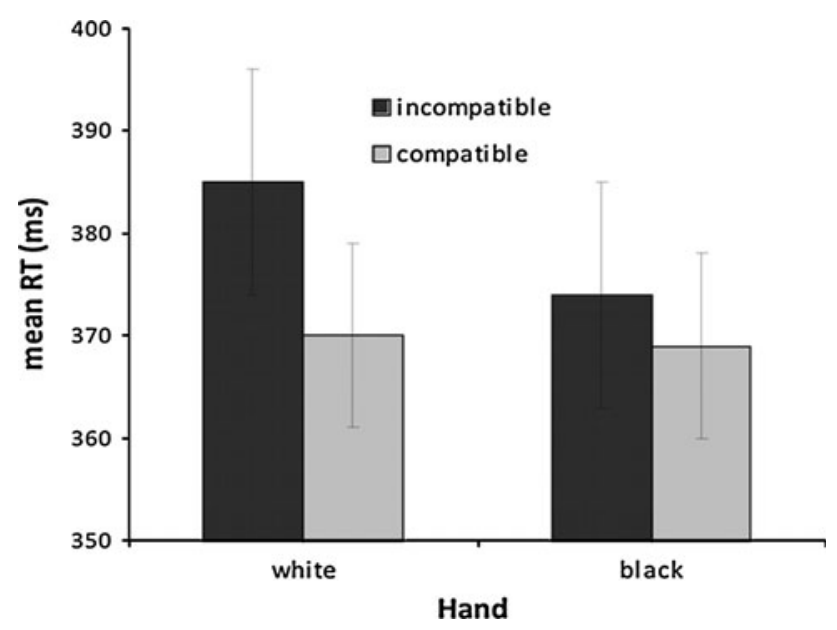

Fig. 2 Mean reaction times of the joint Simon task as a function of interaction partner (white vs. black) and compatibility (compatible vs. incompatible trials)

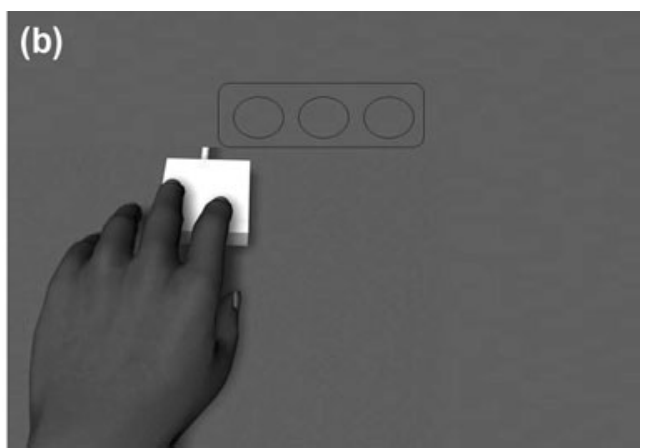

in-group member, no compatibility effect was obtained for interactions with a black out-group member. Thus, actions were co-represented only when both actor and co-actor belonged to the same group, thereby supporting findings demonstrating that higher-order processes can influence action co-representation (Hommel et al. 2009; Müller et al. 2011). In study II, we explored how flexible these biases are by instructing participants to take the perspective of an out-group member before interacting with another member of the same out-group. We predicted that by taking the perspective of an out-group member the lack of compatibility effects in interactions with black out-group members would be eliminated.

\section{Study II}

Research on stereotypes demonstrated that both the expression and accessibility of stereotypes for out-group members decrease after perspective taking (Galinsky and Moskowitz 2000), and that taking the perspective of an outgroup member results in more positive attitudes towards out-group members (Batson et al. 1997; Batson et al. 2002). For example, people are more likely to ascribe selfdescriptive traits onto a target person after taking that person's perspective (Davis et al. 1996). Moreover, perspective takers better understand the actions of others (Lozano et al. 2006). Galinsky et al. (2005) suggested that these positive consequences of perspective-taking result from increased self-other overlap in cognitive representations (see also Davis et al. 1996). Based on these findings, we hypothesised that taking the perspective of an out-group member would lead to stronger co-representation of the out-group member's actions. In the second study, white participants read a story about either a white in-group member or a black out-group member (a Surinamese man, member of a stigmatised immigrant group in the Netherlands; Verkuyten and Thijs 2002) and were asked to take the perspective of the main character, before performing a joint Simon task with a white or black co-actor (displayed as a hand on the monitor). 


\section{Methods}

\section{Participants}

Forty-three white undergraduate students from the Radboud University Nijmegen participated in the study (37 women; 18-31 years). They received course credit or financial compensation for their participation. All participants were right-handed and had normal to corrected-tonormal vision. Participants were randomly assigned to one of the two experimental conditions. None of the participants took part in study $\mathrm{I}$.

\section{Experimental design and procedure}

The experiment had a 2 (story: in-group vs. out-group) $\times 2$ (hand colour: white vs. black) $\times 2$ (compatibility: compatible vs. incompatible) mixed model design, with story as between-subjects factor, and hand colour and compatibility as within-subjects factors.

Participants read a short story about a law student and were instructed to take the perspective of the main character while reading. In the 'in-group' condition the main character was introduced as a white student from the Netherlands, in the 'out-group' condition as a black student from Suriname. After reading the story, all participants performed the same joint Simon tasks described in study I, with an animation of either a white hand or a black hand.

\section{Results}

All trials with RTs above $1500 \mathrm{~ms}$ or below $150 \mathrm{~ms}$ (7 trials, $0.4 \%$ ) were excluded from further analysis. Difference scores were calculated by subtracting the average RT of compatible trials from the average RT of incompatible trials. The RTs were log-transformed, but untransformed mean RTs are reported in ms. Difference scores were calculated by subtracting the average RT of compatible trials from the average RT of incompatible trials. One participant with difference scores more than 2.5 SDs above the mean, and two participants with mean RTs were 3 SDs above the mean were excluded from further analysis.

A 2 (story: in-group vs. out-group) $\times 2$ (hand colour: white vs. black) $\times 2$ (compatibility: compatible vs. incompatible) GLM-repeated measures analysis revealed a significant main effect of compatibility: participants were slower on incompatible trials $(M=386$; $\mathrm{SE}=6)$ than on compatible trials $(M=371 ; \mathrm{SE}=5), F(1,38)=27.98$, $P=.001, \quad \eta \rho^{2}=.42$. No effect of hand colour $(F(1,38)=1.28, P=.26)$, or any significant two-way interaction (all $F^{\prime} s<1$ ) were found. As predicted, the three-way interaction was significant, $F(1,38)=4.26$,
$P=.05, \eta \rho^{2}=.10$ (Fig. 3). For both stories, a compatibility effect was obtained with a co-acting white agent, $F(1,38)=18.04, P=.001, \eta \rho^{2}=.32$ (Fig. 3a); Participants were faster for compatible trials than on incompatible trials irrespective of taking the perspective of a in-group member or an out-group member (story in-group: $t(20)=3.43, \quad P=.003$; story out-group: $t(18)=2.60$, $P=.02)$. More importantly, the occurrence of a compatibility effect with a co-acting black agent depended on whether participants took the perspective of an in-group member or out-group member, $F(1,38)=4.11, P=.05$, $\eta \rho^{2}=.10$ (Fig. 3b). There was no compatibility effect for the black agent when participants read the story about an in-group member, $t(20)<1$, n.s. However, a compatibility effect was found when they read the story about an outgroup member, $t(18)=4.14, P=.001$.

\section{General discussion}

In the present research, we investigated whether action corepresentation differs depending on group-membership of an interaction partner and whether this difference could be overcome by taking the perspective of an out-group member. The results of our first study demonstrated that actions of out-group members are co-represented differently than actions of in-group members. While participants co-represent actions only for a white in-group member, they did not co-represent actions of a black out-group member. In the second study, co-representation of an outgroup member's action could be induced by instructing participants to take the perspective of an out-group member before performing the Simon task. Participants taking the perspective of an in-group member still only co-represented actions of a white, but not of a black interaction partner. However, participants who first took the perspective of an out-group member showed compatibility effects when interacting with both a white agent and a black agent.

Recent research showed that higher-order processes have a strong influence on whether people co-represent actions of others or not (Gutsell and Inzlicht 2009; Hommel et al. 2009; Müller et al. 2011). In line with these findings, we demonstrate that actions of out-group members are indeed co-represented substantially less than actions performed by in-group members. Depending on social factors like group-membership, the overlap between representations of one's own actions and representation of actions of others is reduced. However, these differences in action co-representation are not stable but flexible and can be eliminated by increasing perspective taking between members from different social groups. Whereas, prior literature already demonstrated a modulation of error-related brain activity during observation of actions performed by 
Fig. 3 Mean reaction times of the joint Simon task during interaction with a white agent (a) and a black agent (b) as a function of story (in-group member vs. out-group member) and compatibility (compatible vs. incompatible trials)
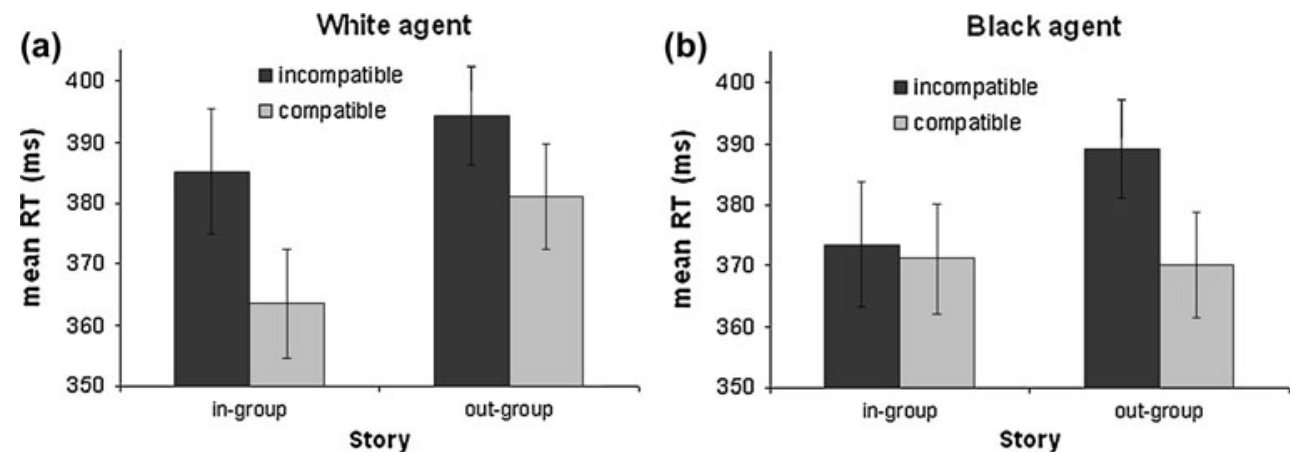

in-group vs. out-group members in a competitive context (Newman-Norlund et al. 2009), and differences in motor cortex activation during observation of in-group vs. outgroup members' actions (Gutsell and Inzlicht 2009), the present findings extend these results by using different methods, and showing the same modulatory effect in the absence of a competitive context.

Interestingly, it does not seem necessary to take the perspective of a specific out-group member for action corepresentation to occur, but sufficient to take the perspective of any member of the out-group, as demonstrated in our second study. Indeed, previous literature demonstrated that the positive consequences of taking the perspective of one out-group member are generalised to the whole outgroup (Aron et al. 1991; Batson et al. 1997). After taking the perspective of an out-group member, attitudes towards the out-group become more positive, and these changes in attitude can facilitate actual helping behaviour towards any member of the out-group (Batson et al. 2002).

Perspective taking increases both positivity towards an out-group, as well as self-other overlap with an out-group interaction partner (Batson et al. 1997). This raises the question of whether increased self-other overlap or increased positivity is responsible for our findings. Based on the literature, both explanations are possible; it has been argued that action co-representation partly depends on the perceived overlap of one's representation of the self and the representation of the interaction partner (Müller et al. 2011). In addition, recent research demonstrates that the valence of the interaction influences action co-representation (Hommel et al. 2009). However, a positive relationship with an interaction partner increases self-other overlap (Aron et al. 1991), and it seems difficult to disentangle positivity and self-other overlap as the possible underlying mechanism. Further research is definitely needed to clarify this issue.

Another minor problem of the present research design might be that in-group/out-group membership correlates with a perceptible distinction, i.e. hand colour, so one might argue that our findings are influenced by these differences, and not purely by group membership. Specifically, effects of our first study could be due to the fact that the white hand was perceptually more similar to participants' own hand, which may minimise the compatibility effect during interaction with the black hand. Similarly, in study II, taking the perspective of a black person may prime participants to attend to the hand with a black skin colour. However, recent research suggests that groupmembership and not physical similarity or familiarity is primarily involved in influencing in-group biases (Avenanti et al. 2010). Furthermore, comparable effects of perspective taking have been found in the absence of differences in skin colour (Müller et al. 2011).

Co-representation of our co-actors actions is an essential part in successful interaction (Sebanz et al. 2006), with people tending to more strongly co-represent actions of coactors from the same in-group, with whom one has a positive relationship, and with whom one does not compete (e.g., Hommel et al. 2009). It is possible that to categorise another person as an in-group member might function as an enhanced indication that this person is a co-actor, thereby triggering the tendency to co-represent his or her actions. While we cannot link the present findings to individual differences in prejudice, recent research has shown that individual levels of prejudice influences action co-representation for out-group members, demonstrating that people with higher levels of prejudice co-represent actions less than people with low levels of prejudice (Gutsell and Inzlicht 2009). The authors argue that because of these differences in action co-representation "empathy may be restricted to close others and, without active effort, may not extend to out-groups, making them likely targets of prejudice and discrimination" (page S52). These findings are additionally underpinned by research revealing that corepresentation of pain, as well as emotion recognition is also restricted to in-group members (Avenanti et al. 2010; Elfenbein and Ambady 2002; Xu et al. 2009). The present research is, to the best of our knowledge, the first showing that perspective taking may offer a solution: taking the perspective of an out-group member not only decreases stereotype accessibility and improves attitudes towards the out-group but also improves coordination of actions on a very basic level, thereby improving social interactions. 
Acknowledgments We thank Wesley van Pinxteren and Sarah Opsomer for their help in collecting the data, Jeroen Derks for creating the stimuli, and Jos Wittebrood and Pascal de Water for technical support. This research was supported by a open MaGW grant from the Dutch Research Foundation NWO (400-07-203) awarded to Rick van Baaren. Simone Kühn is a Postdoctoral Fellow of the Research Foundation Flanders (FWO).

Open Access This article is distributed under the terms of the Creative Commons Attribution Noncommercial License which permits any noncommercial use, distribution, and reproduction in any medium, provided the original author(s) and source are credited.

\section{References}

Aron A, Aron EN, Tudor M, Nelson G (1991) Close relationships as including other in the self. J Pers Soc Psychol 60:241-253

Aron A, McLaughlin-Volpe T, Mashek D, Lewandowski G, Wright SC, Aron EN (2005) Including others in the self. In: Stroebe W, Hewstone $M$ (eds) European review of social psychology. Psychology press, Hove, E, Sussex, UK, pp 101-132

Avenanti A, Sirigu A, Aglioti SM (2010) Racial bias reduces empathic sensorimotor resonance with other-race pain. Curr Biol 20:1018-1022. doi:10.1016/j.cub.2010.03.071

Batson CD, Polycarpou MP, Harmon-Jones E, Imhoff HJ, Mitchener EC, Bednar LL et al (1997) Empathy and attitudes: can feeling for a member of a stigmatized group improve feelings toward the group? J Pers Soc Psychol 72:105-118

Batson CD, Chang J, Orr R, Rowland J (2002) Empathy, attitudes, and action: can feeling for a member of a stigmatized group motivate one to help the group? Pers Soc Psychol B 28:1656-1666. doi:10.1177/014616702237647

Brass M, Bekkering H, Prinz W (2001) Movement observation affects movement execution in a simple response task. Acta Psychol 106:3-22

Brewer MB (1979) In-group bias in the minimal intergroup situation: a cognitive motivational analysis. Psychol Bull 86:307-324

Davis MH, Conklin L, Smith A, Luce C (1996) Effects of perspective taking on the cognitive representation of persons: a merging of self and other. J Pers Soc Psychol 70:713-726

Dijksterhuis A, Bargh JA (2001) The perception-behavior expressway: automatic effects of social perception on social behavior. Adv Exp Soc Psychol 33:1-40

Elfenbein HA, Ambady N (2002) On the universality and cultural specificity of emotion recognition: a meta-analysis. Psychol Bull 12:203-235. doi:10.1037//0033-2909.128.2.203

Galinsky AD, Moskowitz GB (2000) Perspective-taking: decreasing stereotype expression, stereotype accessibility, and in-group favouritism. J Pers Soc Psychol 78:708-724. doi:10.1037// 0022-3514.78.4.708

Galinsky AD, Ku G, Wang CS (2005) Perspective-taking and selfother overlap: fostering social bonds and facilitating social coordination. Group Process Interg 8:109-124. doi:10.1177/ 1368430205051060

Gutsell JN, Inzlicht M (2009) The closed circle of empathy: observing outgroups does not result in sensorimotor mu suppression. Psychophysiology 46:S51-S52

Hommel B, Colzato LS, van den Wildenberg WPM (2009) How social are task representations? Psychol Sci 7:794-798

Lozano SC, Hard BM, Tversky B (2006) Perspective taking promotes action understanding and learning. J Exp Psychol Human 32:1405-1421. doi:10.1037/0096-1523.32.6.1405

Müller BCN, Brass M, Kühn S, Tsai C-C, Nieuwboer W, Dijksterhuis A, van Baaren RB (2011) When Pinocchio acts like a human, a wooden hand becomes embodied. Action co-representation for non-biological agents. Neuropsychologia. doi:10.1016/ j.neuropsychologia.2011.01.022

Newman-Norlund RD, Ganesh S, van Shie HT, de Bruijn ERA, Bekkering H (2009) Self- identification and empathy modulate error-related brain activity during the observation of penalty shots between friend and foe. Soc Cogn Affect Neur 4:10-22. doi: $10.1093 / \mathrm{scan} / \mathrm{nsn} 028$

Preston SD, de Waal FBM (2002) Empathy: its ultimate and proximate bases. Behav Brain Sci 25:1-72

Prinz W (1997) Perception and action planning. Euro J Cogn Psychol 9:129-154

Sebanz N, Knoblich G, Prinz W (2003) Representing others' actions: just like one's own? Cognition 8:11-21. doi:10.1016/S0010-0277 (03)00043-X

Sebanz N, Knoblich G, Prinz W (2005) How to share a task: core presenting stimulus-response mappings. J Exp Psychol Human 31:1234-1246. doi:10.1037/0096-1523.31.6.1234

Sebanz N, Bekkering H, Knoblich G (2006) Joint action: bodies and minds moving together. Trends Cogn Sci 10:70-76. doi: 10.1016/j.tics.2005.12.009

Tajfel H, Turner JC (1979) An integrative theory of intergroup conflict. In: Austin WG, Worchel S (eds) The social psychology of intergroup relations. Brooks-Cole, Monterey

Tsai C-C, Brass M (2007) Does the human motor system simulates Pinocchio's actions? Coaction with a human hand versus a wooden hand in a dyadic interaction. Psychol Sci 18:1058-1062

Tsai C-C, Kuo W-J, Jing J-T, Hung DL, Tzeng OJL (2006) A common coding framework in self-other interaction: evidence from joint action task. Exp Brain Res 175:353-362. doi:10.1007/ s00221-006-0557-9

Tsai C-C, Kuo W-J, Hung DL, Tzeng OJL (2008) Action co-representation is tuned to other humans. J Cognitive Neurosci 20:2015-2024

Verkuyten M, Thijs J (2002) Racist victimization among children in The Netherlands: the effect of ethnic group and school. Ethnic Racial Stud 25:310-331. doi:10.1080/01419870120109502

Xu X, Zuo X, Wang X, Han S (2009) Do you feel my pain? Racial group membership modulates empathic neural responses. J Neurosci 29:8525-8529. doi:10.1523/JNEUROSCI.2418-09.2009 